\title{
Transadaptation and Standardization of Tinnitus Functional Index in Bengali
}

\author{
Suraj Kumar ${ }^{1}$, Himanshu Kumar ${ }^{2}$, Indranil Chatterjee ${ }^{3}$, Bibhu Prasad Hota ${ }^{4}$, \\ Akanksha Kumari $^{5}$ \\ ${ }^{I}$ (Assistant Professor in speech and audiology, Pacific medical college and hospital, Rajasthan, India) \\ 2, 3, 4 (Lecturer in Speech and Hearing, AYNIHH, ERC, Kolkata, India) \\ ${ }^{5}$ (Audiologist and speech language pathologist, Kolkata, India)
}

\begin{abstract}
Tinnitus is a phantom sound (ringing of the ears) that affects quality of life for millions around the world and is associated in most cases with hearing impairment. There are numerous Self-rated questionnaire in foreign languages used to assess the negative effects of tinnitus. India being multilingual country, these questionnaires are less effective on Indian population. Hence there is a great need to develop and standardize a self-assessment questionnaire in Indian languages. This study aims at transadapting and standardizing Tinnitus Functional Index (TFI-English) into Bengali language. Validity and reliability measures of the newly developed Tinnitus Functional Index-Bengali (B-TFI) was calculated statistically along with its assessment on sixty tinnitus participants (30 with hearing loss and 30 without hearing loss). A significantly good correlation was found when the results of the tool were compared with THI-Bengali and TFI-English for both the groups of participants. Test-retest reliability showed high correlation between the conditions on all the eight sub-scales of B-TFI. B-TFI is found to be a good tool with high internal consistency for detecting tinnitus related handicap. Study also provides information and need for further research.
\end{abstract}

Keywords: Bengali, Tinnitus, TFI

\section{Introduction}

The term tinnitus comes from the Latin word 'tinnire', which means to ring. Tinnitus is defined by the American National Standards Institute (ANSI, 1969) as "the sensation of sound without external stimulation". Tinnitus can be perceived in one ear, both ears, or in the head. It is usually described as a ringing noise, but in some patients it is perceived as a buzzing, hissing, humming, or whistling sound or as ticking, clicking, roaring, "crickets" or "Locusts", tunes, songs or beeping. It has also been described as a "whooshing" sound, as of winds or waves. Tinnitus is a rising problem in the present society with 10 to $30 \%$ of the adult population suffering from it (Heller, 2000). Tinnitus can be broadly classified into two categories namely objective and subjective tinnitus (McFadden, 1982). Objective tinnitus is noise in the ears of the sufferer which can be heard by an observer as well as by the tinnitus sufferer. Objective tinnitus is produced by a physical source within the body that activates the cochlea by air and/or bone conduction, and could thus also be called somatic sound (Dobie \& Snow, 2004). Subjective tinnitus is a sound in the ear or head which is only heard by sufferer (Hazell, 1987). The tinnitus can occur with or without hearing loss. Up to $90 \%$ of tinnitus patients are also affected by hearing loss (Nicolas-Puel et al., 2001). Tinnitus can be associated with sensorineural, conductive and mixed type of hearing loss. In most of the cases the tinnitus is related to sensorineural type of hearing loss (predominantly sensori loss). In some cases, tinnitus is present due to some kind of middle ear problems. Few researchers have also found neurological aspects of the tinnitus. It is observed that the person having tinnitus has left frontal activation and right frontal activation with negative affect (Davidson et al., 2002). The temporal and left frontal cortex is involved in tinnitus related cortical network as found from a neurophysiological study about distress. The etiology of tinnitus remains unknown, although a number of theories have been proposed. The majority of cases are called idiopathic by researchers and physicians. It is most likely that the disorder is heterogeneous (Moller, 1997).Other causes of tinnitus include other ear problems, chronic health conditions, and injuries or conditions that affect the nerves in the ear or the hearing center in our brain. Other etiologies are an age-related hearing loss, exposure to loud noise, earwax blockage, ear bone changes, meniere's disease, temperomandibular joint disorders, head injuries or neck injuries, and acoustic neuroma. Tinnitus can lead to a wide range of complaints in several areas of functioning. People experience different reactions to tinnitus and may have mild to severe consequences that have an impact on an individual's daily activities (Heller, 2000). One of the most common psychopathologies clinically observed in tinnitus patients is depression (Johnson, 1982). Feelings of hopelessness, self-pity, inadequacy, along with a dependence on others to solve problems, withdrawal from social situations, and frequency of other illnesses are examples of these depression-related states. Some tinnitus patients have committed suicide to escape the noise. The impact of these effects may cause an individual to 
perceive a reduction in his/her quality of life. Chronic sleep deprivation caused by tinnitus, poses an effect on attention, and as a result, an effect on cognition giving rise to associated frustration, anger and as a whole a disturbed emotional status. These kind of effects can be self-perpetuating, with the potential to affect every aspect of life. The assessment of tinnitus is a challenging task for the audiologist. The assessment of tinnitus consists of two processes. The first involves a complete medical evaluation and second involves an audiometric testing. Most importantly an audiometric test should be completed, which includes an audiological assessment, psychoacoustic measure of tinnitus, and measure of loudness discomfort level (LDLs) (Jastreboff, 2000). Basic tinnitus measurements (loudness and pitch matching, minimum masking level, and residual inhibition) were advocated as necessary components of a standardized tinnitus evaluation (Vernon \& Fenwick, 1984). Results from all the audiological tests can help identify the best management procedure for an individual with tinnitus. Another important way to assess tinnitus is with the help of various questionnaires as tinnitus is a subjective symptom. Questionnaires can be both written and verbal and are useful in the assessment process (Henry \& Zaugg, 2005). Self-report questionnaires elucidate the effect that tinnitus has on the individual's daily life. Numerous questionnaires have been developed for scaling the negative impact (severity) of tinnitus, Tinnitus Severity Grading (Coles et al., 1992), Subjective Tinnitus Severity Scale Tinnitus Questionnaires (Halford \& Anderson, 1991), Tinnitus Handicap Questionnaires (Kuk et al., 1990), Tinnitus Handicap Inventory (Newman et al., 1996), Tinnitus Severity Scale (Sweetow \& Levy, 1990), Tinnitus Reaction Questionnaires (Wilson et al., 1991). Existing questionnaires have proven useful for measuring individual differences regarding tinnitus severity.

The Tinnitus Functional Index (TFI) is the most recently developed as well as one of the most used questionnaires worldwide. It was developed by Meikle et al. in 2012. The Tinnitus Functional Index (TFI) is the first tinnitus questionnaire documented for responsiveness, and has the potential to become the new standard for evaluating the effects of intervention for tinnitus, with clinical patients and in research studies. The TFI consists of 8 subscales which address 8 important domains of tinnitus' negative impact: Intrusive (unpleasantness, intrusiveness, and persistence), Sense of control (reduced sense of control), Cognitive (cognitive interference), Sleep (sleep disturbance), Auditory (auditory difficulties attributed to tinnitus), Relaxation (interference with relaxation), Quality of Life (QOL) (quality of life reduced) and Emotional (emotional distress). Each subscale has a brief title (in capital letters) and a 1- or 2-letter abbreviation (e.g. I for Intrusive, SC for Sense of Control). Each of the 8 subscales consists of 3 items except for the Quality of life subscale, which consists of 4 items. The Tinnitus Functional Index (TFI) consists of 25 questions ranked using a 0-10 points rating scale where the subject has to rate, perceptually, the loudness of his or her tinnitus. The Tinnitus Functional Index (TFI) has been used for scaling the severity and negative impact of tinnitus during intake assessment and for measuring treatment-related changes in tinnitus (responsiveness). It provides comprehensive coverage of multiple tinnitus severity domains. Self-rated questionnaires are important for the assessment of tinnitus but most of them are in foreign languages. Such questionnaires when used with the person having different language backgrounds will not yield an exact result. Therefore, a new instrument may be developed or an existing instrument may be translated into another language (Guillemin, Bombardier, \& Beaton, 1993). In Indian context, due to a huge linguistic diversity, for obtaining reliable assessment results of any questionnaire there is a need to develop a questionnaire in regional languages. Questionnaires are mostly available in English, so the present study tried to provide a linguistic specific questionnaire to measure the negative effect of tinnitus in the Indian population. The present study attempts to transadapt and standardize Tinnitus Functional Index (TFI) in the Bengali language so it will help provide an efficient and easy measure of negative impact related with tinnitus clinically for the Bengali speaking population.

\section{Participants}

\section{Methodology}

A total of 60 native Bengali speaking participants (mean age: 48.1, standard deviation of age: 14.01) having subjective, permanent and spontaneous (not occurring only during exposure to noise or immediately after) unilateral or bilateral subjective idiopathic tinnitus with hearing loss (mild to moderate sensorineural) and without hearing loss were taken in the present study.

Group-1: consisted of 30 Bengali speaking participants (mean age: 47.83, standard deviation of age: 16.21) having tinnitus with mild to moderate hearing loss of age range 20 to 70 years. Group 1 was again divided into two sub-groups of 15 male and 15 female participants.

Group-2: consisted of 30 Bengali speaking participants (mean age: 45.53, standard deviation of age: 16.23) having tinnitus with normal hearing of age range 20 to 70 years. Group 2 was again divided into two sub-groups of 15 male and 15 female participants. 


\section{Inclusion criteria}

The inclusion criteria were assessed through case history and audiological evaluation. Tympanometry was done in order to find out any middle ear pathology of the participants having tinnitus. Otoacoustic emission (OAE) and auditory brainstem response (ABR) were used to screen out any signs of auditory neuropathy in the participants. Loudness discomfort level (LDL) testing was also done to identify the discomfort level of the participants.

Participants were selected on the basis of following inclusionary criteria-

- Participants having subjective unilateral or bilateral tinnitus.

- Participants should not receive any intervening treatment between tests and retest conditions.

- Participants having hearing loss (mild to moderate sensorineural hearing loss) and not having hearing loss.

- Participants without any neurological disorder.

- Participants without any cognitive impairment.

- Participant without any middle ear disorder.

- Participants without any history of acoustic trauma or noise exposure.

- Participants without any current medication.

- Participants should be native speakers of Bengali and also proficient into speaking and reading English language.

\section{Gathering of information from participants}

For recognizing whether participants really have tinnitus or not, tinnitus characteristics were assessed by using Tinnitus Psychoacoustic Assessment (Vernon and Meikle, 1981) in following steps:

\section{Identification of tinnitus ear}

In this step the "tinnitus ear" and "stimulus ear" are recognized. The ear with the loudest or most prominent tinnitus (the "most bothersome" tinnitus) was called the tinnitus ear, and the contralateral ear was called the stimulus ear. For the participants who had symmetrical tinnitus in both of the ears and had normal sensory perception, identification of tinnitus ear was arbitrary.

\section{Pitch matching}

In this step participants were instructed verbally to match the pitch of tinnitus to that of the pitch of the given stimulus (pure tone/noise). This procedure was conducted twice to ensure that the correct pitch identification has been made by the participant. The octave confusion test was further performed to confirm the final pitch. Pitch matched tone was presented in alternation with a tone an octave above and then an octave below during the octave confusion test.

\section{Loudness matching}

After the tinnitus pitch has been determined, the loudness of the tinnitus was matched with the pitch matched tone. The loudness matched threshold was obtained using a $1 \mathrm{~dB}$ step size resolution in both $\mathrm{dBHL}$ and dBSPL.

\section{Minimum masking level}

Minimum masking level (MML) determines the lowest level of Broad band or Narrow band noise (in $\mathrm{dB}$ HL and dB SPL) that renders the participants tinnitus as inaudible (completely "masks" the tinnitus). MML testing was done binaurally for participants with bilateral tinnitus and monaural testing was done for participants with unilateral tinnitus.

\section{Tools/Instrumentation:}

The following tools/instruments were used for the present study -

- Tinnitus Functional Index (TFI) developed by Meikle et al, (2012).

- Transadapted Tinnitus Functional Index (B-TFI) based on International Test Commission Guideline for Test Translation and Adaptation (2013).

- Bengali version of Tinnitus Handicap Inventory (THI) given by Datta (2013).

- Calibrated dual channel diagnostic audiometer (MAICO MA53) with TDH 39 headphone.

- Immittance audiometer (GSI-39 AUTO TYMP).

- Otoacoustic emission (MAICO ERO SCAN).

- Auditory brainstem response (RMS Medulla AD).

\section{Procedure}

The research was carried out in following stages:

Stage 1: Transadaptation of Tinnitus Functional Index (TFI) English to Bengali.

The linguistic validation of the Tinnitus Functional Index developed by Meikle et al, (2012) into the transadapted Tinnitus Functional Index (B-TFI) and its psychometric specification was done with the assistance of a professional linguist through the following steps: 
Translation:

In the first step Tinnitus Functional Index (TFI) English was translated into Bengali by ten native speakers of Bengali, with high levels of proficiency in English and Bengali. The ten native speakers translated the original instrument into Bengali language separately.

Back Translation:

In the second step of linguistic validation, reverse- translation from Bengali to English was done to check the intactness of meaning by another ten native Bengali speakers with high levels of proficiency in the Bengali and English languages. Back translation of the transadapted Tinnitus Functional Index (B-TFI) to English was done to measure the homogeneity of the original version of Tinnitus Functional Index (TFI).

Preparation of final tool:

Transadapted Tinnitus Functional Index (B-TFI) was arranged accordingly. The transadapted Tinnitus Functional Index (B-TFI) questionnaire consisted of 25 questions, which was divided into eight sub-scales. The eight sub-scales addressed eight important domains of negative impact of tinnitus.

Stage 2: Validation of the transadapted Tinnitus Functional Index (B-TFI) by feedback rating

Transadapted Tinnitus Functional Index (B-TFI) was provided to ten native Bengali speaking Speech Language Pathologists (SLPs), having at least two years of clinical experience for feedback rating and appropriateness of the newly developed tool.

Stage 3: Participant selection

The participants fulfilling the inclusion criteria were selected from the Audiological Department of AYJNIHH, ERC, Kolkata for the study. Verbal consent was also obtained from each participant who partook in the study. Pilot study

The developed transadapted Tinnitus Functional Index (B-TFI) was administered on two participants having tinnitus with hearing loss and two participants having tinnitus without hearing loss. These participants were not included in the actual study. The suggestions given by these participants regarding the test material were included in the actual testing material.

Stage 4: Administering the transadapted test to participants with tinnitus and obtaining the test scores:

The transadapted tinnitus functional index in Bengali was given to all the participants of the present study for self rating. Participants were asked to read the questions carefully and circle the appropriate rating on a 0-10 rating scale. Each rating option was explained to all the participants with the help of few examples from day to day life. Any clarification requested by the participants was provided by the researcher. Time provided to each participant was 40 minutes for completing his or her rating.

Scoring

Scoring of the B-TFI Questionnaire was given on a 0-10 point Likert rating scale where " 0 " was indicative of "least severity" and a score of "10" was indicative of "most severity". Overall B-TFI scores were calculated by summing up all valid answers from the set of questions present in B-TFI (maximum possible score $=250$ if the respondent were to rate all $25 \mathrm{TFI}$ item at the maximum value of 10). Next the respondents mean item scores was obtained by dividing the overall B-TFI score by the number of valid answers then finally it was multiplied by 10 to get the participants overall B-TFI score within the range of 0-100.

Stage 5: Checking the reliability of the transadapted Tinnitus Functional Index (B-TFI):

To measure the homogeneity of 25 items in transadapted Tinnitus Functional Index (B-TFI), inter-item correlation was done. To check test-retest reliability of the transadapted Tinnitus Functional Index (B-TFI), after three week from filling the first B-TFI, the Questionnaire was filled again by the same participant. The retest was done without informing the participant's score of the previous test. No intervening treatment was done between tests and retests condition like psychological management, drug trial, any fitting of hearing aid or noise generator devices. The basic raw data that was obtained from the participants was fed into Microsoft Office Excel 2010, and then Pearson Correlation Coefficients were done by using SAS 9.2 version software.

\section{Validity Assessment}

\section{Result And Discussion}

In computational science and statistics, the term validity refers to whether or not the test measures what it claims to measure (Carmines \& Zeller, 1979). To establish construct validity, back translated Tinnitus Functional Index was compared with the original version of Tinnitus Functional Index (Meikle et al., 2012) by ten speech language pathologists. The validation criteria was based on a three point rating scale $(1=$ Not appropriate, $2=$ Appropriate, $3=$ Mostly appropriate). None of the judge among the ten judges gave a rating of 1 (1=not appropriate) hence the inter judge agreement was calculated between appropriate and most appropriate rating by using Pearson Correlation Coefficients. 
Table 1: Inter judge agreement

\begin{tabular}{|l|l|l|l|l|}
\hline Variable & $\begin{array}{l}\text { N } \\
\text { (No. of judge) }\end{array}$ & Mean & Std. deviation & $\begin{array}{l}\text { R } \\
\text { (corr. Coefficient) }\end{array}$ \\
\cline { 1 - 4 } Appropriate & \multirow{2}{*}{10} & 12.50 & 5.91 & \multirow{2}{*}{-1.00} \\
\cline { 1 - 3 } & \multicolumn{1}{|l}{ Most appropriate } & 12.50 & 5.91 & \\
\hline
\end{tabular}

The result showed good inter judge agreement $(\mathrm{r}=-1.00)$ between the appropriate and most appropriate rating given by the ten judges.

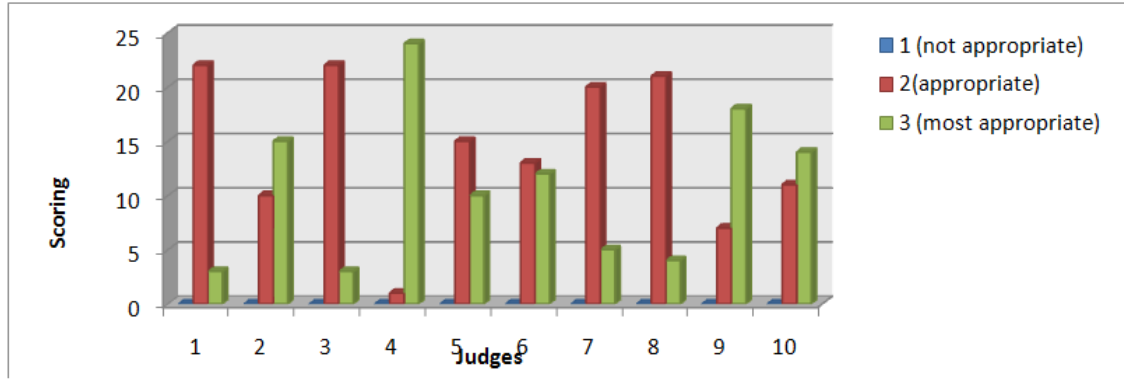

Figure 1: graphical representation showing linguistic validation of the transadapted Tinnitus Functional Index (B-TFI) among ten speech language pathologists (judges).

\section{Discriminate validity}

Pearson Correlation Coefficients was used to correlate the scores of transadapted Tinnitus Functional Index (B-TFI) with Tinnitus Handicap Inventory (THI)-Bengali and also to correlate the scores of transadapted Tinnitus Functional Index (B-TFI) among participants with and without hearing loss having tinnitus.

Table 2: Correlation of the transadapted Tinnitus Functional Index (B-TFI) with Tinnitus handicap inventory (THI)-Bengali.

\begin{tabular}{|l|l|l|l|c|}
\hline Variable & N (No. of sample) & Mean & Std. deviation & $\begin{array}{l}\text { R(corr. } \\
\text { Coefficient) }\end{array}$ \\
\cline { 1 - 3 } THI-Bengali & 60 & 56.00 & 12.29 & \multirow{2}{*}{0.85} \\
\hline B-TFI & 60 & 41.93 & 9.07 & \\
\hline
\end{tabular}

Table 3: Correlation of the transadapted Tinnitus Functional Index (B-TFI) score of participant with hearing loss and without hearing loss having tinnitus.

\begin{tabular}{|l|l|l|l|l|}
\hline $\begin{array}{c}\text { B-TFI } \\
\text { Variable }\end{array}$ & $\begin{array}{l}\text { N (No. Of } \\
\text { Sample) }\end{array}$ & Mean & Std. Deviation & $\begin{array}{l}\text { R(corr. } \\
\text { Coefficient) }\end{array}$ \\
\hline With hearing loss & 30 & 118.46 & 18.60 & \multirow{2}{*}{-0.05} \\
\hline Without hearing loss & 30 & 89.33 & 16.57 & \\
\hline
\end{tabular}

Pearson Correlation Coefficients result explained that there was significantly good correlation between $\mathrm{B}-\mathrm{TFI}$ and THI-Bengali $(\mathrm{r}=0.85$ and $\mathrm{p}$ value $=<.0001)$. Results indicated that there was no significant correlation between the scores of participant with hearing loss and without hearing loss, having tinnitus $(r=-0.05$ and $p$ value $=0.75)$ as shown in table 3.

\section{Concurrent validity}

Pearson Correlation Coefficients was used to correlate the scores of transadapted Tinnitus Functional Index (B-TFI) with Tinnitus Functional Index (TFI)-English in participants with hearing loss and without hearing loss having tinnitus.

Table 4: Correlation of transadapted Tinnitus Functional Index (B-TFI) with Tinnitus Functional Index (TFI)English in participant with hearing loss having tinnitus.

\begin{tabular}{|l|l|l|l|l|}
\hline $\begin{array}{l}\text { With Hearing loss } \\
\text { Variable }\end{array}$ & N (No. of sample) & Mean & $\begin{array}{l}\text { Std. } \\
\text { Deviation }\end{array}$ & $\begin{array}{l}\text { R (corr. } \\
\text { Coefficient) }\end{array}$ \\
\hline TFI-English & 30 & 120.46 & 18.60 & 0.96 \\
\hline B-TFI & 30 & 117.16 & 17.71 & \\
\hline
\end{tabular}


Table 5: Correlation of transadapted Tinnitus Functional Index (B-TFI) with Tinnitus Functional Index (TFI)-

English in participant without hearing loss having tinnitus.

\begin{tabular}{|l|l|l|l|l|}
\hline $\begin{array}{l}\text { Without Hearing loss } \\
\text { Variable }\end{array}$ & N (No. of sample) & Mean & $\begin{array}{l}\text { Std. } \\
\text { Deviation }\end{array}$ & $\begin{array}{l}\text { R corr. } \\
\text { Coefficient) }\end{array}$ \\
\hline TFI-English & 30 & 92.06 & 16.84 & \multirow{2}{*}{0.94} \\
\hline B-TFI & 30 & 89.53 & 15.54 & \\
\hline
\end{tabular}

Pearson Correlation Coefficients results explained that there was a significantly high correlation $(\mathrm{r}=.96$ and $\mathrm{p}$ value $=<.0001)$ when scores of B-TFI and TFI-English was compared in participant with hearing loss having tinnitus. Results also explain that there was significant high correlation $(\mathrm{r}=0.94$ and $\mathrm{p}$ value $=<.0001)$ when scores of B-TFI and TFI-English was compared in participant without hearing loss having tinnitus.

\section{Construct validity}

Pearson Correlation Coefficients was used to correlate the scores of transadapted Tinnitus Functional Index (B-TFI) with matched pitch, loudness and minimum masking level (MML).

Table 6: Correlation of transadapted Tinnitus Functional Index (B-TFI) with matched pitch.

\begin{tabular}{|l|l|l|l|l|}
\hline Variable & N (No. of sample) & Mean & Std. Deviation & R (corr. Coefficient) \\
\cline { 1 - 4 } Pitch & 60 & 4019 & 3160 & \multirow{2}{*}{0.01} \\
\hline B-TFI & 60 & 103.35 & 21.61 & \\
\hline
\end{tabular}

Table 7: Correlation of transadapted Tinnitus Functional Index (B-TFI) with loudness.

\begin{tabular}{|l|l|l|l|l|}
\hline Variable & N (No. of sample) & Mean & Std Deviation & R (corr. Coefficient) \\
\hline Loudnes & 60 & 41.91 & 12.85 & \multirow{2}{*}{0.72} \\
\hline B-TFI & 60 & 103.35 & 21.61 & \\
\hline
\end{tabular}

Table 8: Correlation of transadapted Tinnitus Functional Index (B-TFI) with minimum masking level (MML).

\begin{tabular}{|l|l|l|l|l|}
\hline Variable & N (No. of sample) & Mean & Std. Deviation & $\begin{array}{l}\mathbf{R} \\
\text { Coefficient) }\end{array}$ \\
\hline MML & 60 & 47.83 & 12.85 & \multirow{2}{*}{ (corr. } \\
\hline B-TFI & 60 & 103.35 & 21.61 & 0.67 \\
\hline
\end{tabular}

The results explained that there was significant correlation between the B-TFI and matched loudness $(\mathrm{r}=0.72$, $\mathrm{p}$ value $=<.0001)$ and with minimum masking level $(\mathrm{MML})$ for tinnitus $(\mathrm{r}=0.67, \mathrm{p}$ value $=<.0001)$ but in the case of matched pitch $(\mathrm{r}=0.01, \mathrm{p}$ value $=89)$ results indicated correlation was very poor. Meikle et al. (2012) developed Tinnitus Functional Index (TFI), a self-report tinnitus handicap measure in English. For validity of final twenty-five item questionnaire of Tinnitus Functional index, they had compared the TFI scores with Tinnitus Handicap inventory (THI) and the Visual Analog Scale (VAS). Discriminate validity was measured by comparing the scores of TFI with Beck Depression Inventory-Primary Care (BDI-PC). High Pearson correlations were found between TFI scores and THI scores $(r=0.86)$ and severity on VAS $(r=0.74)$. In addition, the final TFI scores exhibited a moderate correlation $(r=0.5)$ with the BDI-PC. Correlation between audiometric data, psychotic and acoustic measures associated with subjective tinnitus was assessed by Karatas \& Deniz in 2012. They found significant correlation between Visual Analog Scale (VAS) scores and loudness discomfort level, minimum masking level and residual inhibition $(\mathrm{P}>0.05)$. They did not find statistically significant correlation between audiometric data and Tinnitus Handicap Inventory (THI) and Beck Depression Inventory (BDI). Fackrell et al. (2013) had measured Convergent and discriminant validity of the Tinnitus Functional Index. Acceptable convergent and discriminant validity was demonstrated by the high correlations between the score on the Tinnitus Functional Index and Tinnitus Handicap Inventory $(\mathrm{r}=0.82)$ and Tinnitus Handicap Questionnaire ( $\mathrm{r}=0.82)$, moderate correlations with Visual Analogue Scale of Loudness $(\mathrm{r}=0.46)$, Percentage Rating of Annoyance $(\mathrm{r}=0.58)$, Beck's Depression Inventory( $\mathrm{r}=0.57)$, Beck's Anxiety Inventory $(\mathrm{r}=0.38)$ and World Health Organisation Quality of Life Brief(r=-0.48). For validity of Dutch version of Tinnitus Functional Index Rabau, Wouters, \& Heyning (2014) had measured Spearman correlation with the percentage of time aware of the tinnitus and visual analogue scales (VAS) for maximum tinnitus loudness and mean tinnitus loudness. Factor structure was also assessed using exploratory analysis with oblique rotation and compared with the original questionnaire. The result showed that original eight-factor structure could be confirmed in the Dutch version of the TFI. Convergent validity showed good results. Statistically significant correlations were found with the VAS for maximum loudness and for mean loudness.

The construct validity of Danish version of Tinnitus Handicap Inventory was assessed using tinnitus symptom rating scales, Beck Depression Inventory (BDI), State-Trait Anxiety Inventory (STAI), Tinnitus Coping Style Questionnaire (TCSQ), Eysenck Personality Questionnaire (EPQ), and perceived tinnitus loudness and pitch by using Pearson product-moment correlations. Result of the study indicated low non-significant 
correlations between tinnitus pitch $(\mathrm{r}=0.27)$ and loudness $(\mathrm{r}=0.15)$. The Tinnitus Handicap Questionnaire developed by Kuk et al. (1990) was administered to patient who provided subjective loudness ratings (on a scale from 1-100) and psychoacoustic measures. There was a moderately high correlation between the subjective loudness ratings and the degree of tinnitus handicap $(\mathrm{r}=.57$ and $\mathrm{p}=.0001)$ but a low correlation between the loudness matches and tinnitus handicap questionnaire $(r=.27$ and $\mathrm{p}=.04)$. The authors suggested that the psychoacoustic measures and the subjective ratings did not test the same attributes of tinnitus.

\section{Reliability}

Reliability is used to describe the overall consistency of a measure. In the present study internal consistency and test-retest reliability was measured to identify the reliability.

\section{Internal consistency}

Internal consistency of the tool was assessed by using Pearson Correlation Coefficients and the results were given in below table:

Table 9: Inter-item correlation of transadapted Tinnitus Functional Index (B-TFI).

\begin{tabular}{|l|l|l|l|l|l|l|}
\hline Variable & No. item & \multicolumn{2}{|l|}{ Test condition } & Retest condition & R(corr. Coefficient) \\
\hline \multirow{2}{*}{ B-TFI } & \multirow{2}{*}{25} & Mean & Std. deviation & Mean & Std. deviation & \multirow{2}{*}{0.97} \\
\cline { 2 - 6 } & 246.44 & 91.06 & 245.56 & 89.99 & \\
\hline
\end{tabular}

The result indicates the questionnaire of B-TFI has significantly high internal consistency $(\mathrm{r}=0.97$ and $\mathrm{P}$ value $=$ $<.0001)$

\section{Test-retest reliability}

Esposito (2002) has suggested that to assess reliability more precisely, test retest reliability is most appropriate. The test-retest reliability was done on the basis of subscales. Test-retest reliability was calculated by using Pearson Correlation Coefficients.

Table 10: Test-retest reliability of intrusive (unpleasantness, intrusiveness, persistence) sub scale.

\begin{tabular}{|l|l|l|l|l|}
\hline Variable & No. of item & Mean & Std. deviation & R(corr. Coefficient) \\
\hline Test condition & 3 & 369.33 & 104.58 & 0.99 \\
\hline $\begin{array}{l}\text { Retest condition } \\
\text { (after 21dys) }\end{array}$ & 3 & 370.33 & 116.90 & \\
\hline
\end{tabular}

The result explains that there was significantly high correlation $(\mathrm{r}=0.99)$ between the review of participants having tinnitus in test condition (response before twenty-one days) and the retest condition (responses after twenty-one days) response for the Intrusive sub-scale.

Table 11: Test-retest reliability of sense of control (reduced sense of control) sub-scale.

\begin{tabular}{|l|l|l|l|l|}
\hline Variable & N. of item & Mean & Std. deviation & R(corr. Coefficient) \\
\hline Test condition & 3 & 300.33 & 18.77 & 0.80 \\
\hline $\begin{array}{l}\text { Retest condition } \\
\text { (after 21dys) }\end{array}$ & 3 & 295.00 & 10.81 & 0. \\
\hline
\end{tabular}

The result explains that there was significantly good correlation $(r=0.80)$ between the review of participants having tinnitus in test condition (response before twenty-one days) and the retest condition (responses after twenty-one days) response for the sense of control sub-scale

Table 12: Test-retest reliability of cognitive (cognitive interference) sub-scale.

\begin{tabular}{|l|l|l|l|l|}
\hline Variable & No. of Item & Mean & Std. deviation & R(corr. Coefficient) \\
\hline Test condition & 3 & 248.66 & 35.64 & \multirow{2}{*}{0.97} \\
\cline { 1 - 3 } $\begin{array}{l}\text { Retest condition } \\
\text { (after 21dys) }\end{array}$ & 3 & 243.66 & 28.02 & \\
\hline
\end{tabular}

The result explains that there was significantly high correlation $(\mathrm{r}=0.97)$ between the review of participants having tinnitus in test condition (response before twenty-one days) and the retest condition(responses after twenty-one days) response for the cognitive sub-scale.

Table 13: Test-retest reliability of sleep (sleep disturbance) sub-scale.

\begin{tabular}{|l|l|l|l|l|}
\hline Variable & No. of Item & Mean & Std. deviation & R(corr. Coefficient) \\
\hline Test condition & 3 & 105.33 & 15.01 & \multirow{2}{*}{0.99} \\
\hline $\begin{array}{l}\text { Retest condition } \\
\text { (after 21dys) }\end{array}$ & 3 & 113.66 & 16.25 & \\
\hline
\end{tabular}


The result explains that there was significantly high correlation $(\mathrm{r}=0.99)$ between the review of participants having tinnitus in test condition (response before twenty-one days) and the retest condition (responses after twenty-one days) response for the sleep sub-scale.

Table 14: Test-retest reliability of auditory (auditory difficulties attributed to tinnitus) sub-scale.

\begin{tabular}{|l|l|l|l|l|}
\hline Variable & No. of Item & Mean & Std. deviation & R(corr. Coefficient) \\
\hline Test condition & 3 & 188.00 & 33.46 & \multirow{2}{*}{0.94} \\
\cline { 1 - 4 } $\begin{array}{l}\text { Retest condition } \\
\text { (after 21dys) }\end{array}$ & 3 & 193.66 & 34.15 & \\
\hline
\end{tabular}

The result explains that there was significantly high correlation value is $(r=0.94)$ between the review of participants having tinnitus in test condition (response before twenty-one days) and the retest condition (responses after twenty-one days) response for the auditory sub-scale.

Table 15: Test-retest reliability of relaxation (interference with relaxation) sub-scale.

\begin{tabular}{|l|l|l|l|l|}
\hline Variable & No. of Item & Mean & Std. deviation & R(corr. Coefficient) \\
\hline Test condition & 3 & 194.00 & 13.60 & \multirow{2}{*}{0.99} \\
\hline $\begin{array}{l}\text { Retest condition } \\
\text { (after 21dys) }\end{array}$ & 3 & 197.66 & 11.71 & \\
\hline
\end{tabular}

The result explains that there was significantly high correlation (0.99) between the review of participants having tinnitus in test condition (response before twenty-one days) and the retest condition (responses after twenty-one days) response for relaxation sub-scale.

Table 16: Test-retest reliability of quality of life (quality of life reduced) sub-scale.

\begin{tabular}{|l|l|l|l|l|}
\hline Variable & No. of Item & Mean & Std. deviation & R(corr. Coefficient) \\
\hline Test condition & 3 & 221.75 & 39.96 & \multirow{2}{*}{0.88} \\
\cline { 1 - 3 } $\begin{array}{l}\text { Retest condition } \\
\text { (after 21dys) }\end{array}$ & 3 & 212.50 & 36.75 & \\
\hline
\end{tabular}

The result explains that there was significantly high correlation $(r=0.88)$ between the review of participants having tinnitus in test condition (response before twenty-one days) and the retest condition (responses after twenty-one days) response for the quality of life sub-scale.

Table 17: Test-retest reliability of emotional (emotional distress) sub-scale.

\begin{tabular}{|l|l|l|l|l|}
\hline Variable & No. of Item & Mean & Std. deviation & \multirow{2}{*}{ R(corr. Coefficient) } \\
\cline { 1 - 4 } Test condition & 3 & 352.33 & 11.15 & \multirow{2}{*}{0.96} \\
\hline Retest condition & 3 & 349.00 & 13.52 & \\
\hline
\end{tabular}

The result explains that there was significantly high correlation $(r=0.96)$ between the review of participants having tinnitus in test condition (response before twenty-one days) and the retest condition (responses after twenty-one days) response for the emotional sub-scale. Chandra (2013) had done the internal consistency reliability, test-retest reliability, convergent and divergent validity of Tinnitus Functional Index (TFI) to be used in New Zealand. They had obtained convergent validity by correlating the overall scores of Tinnitus Functional Index (New Zealand) with Tinnitus Handicap Questionnaire (THQ). The results indicated significant correlation between Tinnitus Functional Index (New Zealand) and THQ $(r=.71$ and $p=<.001)$. They had performed test and retest reliability on the basis of sub-scale, which showed statistically significant correlation between test and retest condition of all eight sub-scale of New Zealand version of Tinnitus Functional Index (intrusive $r=0.83$, sense of control $r=0.63$, cognitive $r=0.72$, sleep $r=0.87$, auditory $r=0.80$, relaxation $r=0.61$, Quality of life $r=0.75$, emotional $r=0.72$ ). They had also assessed internal consistency on the basis of overall score indicating significantly high internal consistency $(r=0.97)$ of New Zealand version of Tinnitus Functional Index (TFI).

Mahmoudian et al. (2011) has validated the Persian version of the Tinnitus Handicap Inventory (THIP). They have used translation and back translation for linguistic validation of the original version of THI into Persian version (THI-P). Results of this study indicate adequate test-retest reliability for the THI-P $(r=0.96)$ and also indicated adequate internal consistency of the THI-P $(r=0.943)$ with a total item correction $(r=0.939)$ indicating its reproducibility. Internal Consistency and test-retest reliability of United Kingdom (UK) version of Tinnitus Functional Index (TFI) was assessed by Fackrell et al in 2013. They have assessed test retest reliability on the basis of subscale, result indicate statistically significant correlation between test and retest condition of all eight sub-scale (intrusive $r=0.83$, sense of control $r=0.75$, cognitive $r=0.66$, sleep $r=0.78$, auditory $r=0.90$, relaxation $\mathrm{r}=0.67$, Quality of life $\mathrm{r}=0.63$, emotional $\mathrm{r}=0.76$ ). Results also show significant high internal consistency $(\mathrm{r}=0.97)$. 


\section{Summary And Conclusion}

There are very few questionnaires developed in Indian languages to assess negative impact due to tinnitus. The present study was an attempt towards transadapting and standardizing Tinnitus Functional Index (TFI) in the Bengali language. It will provide an efficient and easy measure of negative impact related with tinnitus clinically for the Bengali speaking population. To standardize Tinnitus Functional Index (TFI) in Bengali, internal consistency and reliability of the B-TFI were thoroughly studied after the linguistic validation. Discriminate validity, Concurrent validity and Construct validity to standardize the B-TFI. The results indicated good correlation between score of THI- Bengali and B-TFI, high internal consistency among the twenty-five questionnaire of B-TFI and high correlation in test-retest reliability. The standardized outcome measures would facilitate comparisons across treatments and allow for meta-analyses and summarizing results across treatment. The present results will facilitate the provision of evidence-based treatment decisions for people with tinnitus.

\section{References}

[1]. American National Standards Institute. (1969). Specification for Audiometers. ANSI S3.6. New York: American National Standards Institute.

[2]. Carmines, E. G., \& Zeller, R. A. (1979). Reliability and validity assessment (Vol. 17). Sage publications.

[3]. Coles, R. R. A., Lutman, M. E., Axelsson, A., et al. (1992). Tinnitus Severity Gradings: Cross-Sectional Studies. In J. M. Aran \& R. Dauman (Eds). Proceedings of the Fourth International Tinnitus Seminar (453-455). New York, NY: Kugler Publications.

[4]. Datta, P. (2013). Development and Standardization of Tinnitus Handicap Inventory in Bengali. Unpublished Dissertation. Master of Audiology and Speech Language Pathology. The West Bengal University of Health Sciences.

[5]. Davidson, R. J., Pizzagalli, D., Nitschke, J. B., \& Putnam, K. (2002). Depression: perspectives from affective neuroscience. Annual review of psychology, 53(1), 545-574.

[6]. Dobie, R. A., \& Snow, J. B. (2004). Overview: suffering from tinnitus. Tinnitus: Theory and management (pp 1-7). Lewiston, NY: BC Decker.

[7]. Esposito, J. L. (2002). Interactive, multiple-method questionnaire evaluation research: A case study. Paper presented at International Conference in questionnaire development, Evaluation and Testing (QDET) Methods. Charleston, SC.

[8]. Fackrell, K., Hall, D. A., Barry, J. G., \& Hoare, D. J. (2013). UK validation of the Tinnitus Functional Index (TFI): convergent and discriminant validity. 7th international Tinnitus Research Initative Conference. Valencia, Spain.

[9]. Guillemin, F., Bombardier, C., \& Beaton, D. (1993). Cross-cultural adaptation of health-related quality of life measures: literature review and proposed guidelines. Journal of clinical epidemiology, 46(12), 1417-1432.

[10]. Halford, J., \& Anderson, S. D. (1991). Tinnitus severity measured by a subjective scale, audiometry and clinical judgment. The Journal of Laryngology \& Otology, 105(2), 89-93.

[11]. Hazell, J. (1987). Tinnitus. New York, NY: Churchill Livingstone.

[12]. Heller, A. J. (2000). Hearing. New York Academic press.

[13]. Henry, J. A., \& Zaugg, T. L. (2005). Clinical guide of audiological tinnitus management and assessment. American Journal of Audiology, 39, 284-291.

[14]. Karatas, E., \& Deniz, M. (2012). The comparison of acoustic and psychic parameters of subjective tinnitus. European Archives of Oto-Rhino-Laryngology, 269(2), 441-447.

[15]. Kuk, F. K., Tyler, R. S., Russell, D., \& Jordan, H. (1990). The psychometric properties of a tinnitus handicap questionnaire. Ear and hearing, 11(6), 434-445.

[16]. Mahmoudian, S., Shahmiri, E., Rouzbahani, M., Jafari, Z., Reza Keyhani, M., Rahimi, F., \& Farhadi, M. (2011). Persian language version of the "Tinnitus Handicap Inventory": translation, standardization, validity and reliability.International Tinnitus Journal, 16(2), 93-103.

[17]. McFadden, D. (1982). Tinnitus: facts, theories, and treatments. Washington, DC: National Academies Press.

[18]. Meikle, M. B., Henry, J. A., Griest, S. E., Stewart, B. J., Abrams, H. B., McArdle, R., \& Vernon, J. A. (2012). The tinnitus functional index: development of a new clinical measure for chronic, intrusive tinnitus. Ear and hearing, 33(2), $153-176$.

[19]. Moller, A. R. (1997). Similarities between chronic pain and tinnitus. Otology \& Neurotology, 18(5), 577-585.

[20]. Newman, C. W., Jacobson, G. P., \& Spitzer, J. B. (1996). Development of the tinnitus handicap inventory. Archives of Otolaryngology Head \& Neck Surgery, 122(2), 143-148.

[21]. Nicolas-Puel, C., Faulconbridge, R. L., Guitton, M., Puel, J. L., Mondain, M., \& Uziel, A. (2001). Characteristics of tinnitus and etiology of associated hearing loss: a study of 123 patients. International Tinnitus Journal, 8(1), 37-44.

[22]. Rabau, S., Wouters, K., \& Van de Heyning, P. (2013). Validation and translation of the Dutch tinnitus functional index. BENT, 10(4), 251-258.

[23]. Sweetow, R. W., \& Levy, M. C. (1990). Tinnitus severity scaling for diagnostic/therapeutic usage. Hearing Instrument, 41 , 44-46.

[24]. Vernon, J. A., \& Meikle, M. B. (1981). Tinnitus masking: unresolved problems. In CIBA foundation symposium, 85, (239-56).

[25]. Vernon, J., \& Fenwick, J. (1984). Identification of tinnitus: a plea for standardization. The Journal of Laryngology \& Otology, 98(S9), 45-53.

[26]. Wilson, P. H., Henry, J., Bowen, M., \& Haralambous, G. (1991). Tinnitus Reaction Questionnaire: Psychometric Properties of a Measure of Distress Associated With Tinnitus. Journal of Speech Hearing Research, 34(1), 197-201. 